\title{
SYMPOSIUM
}

\section{Neurocognitive predictors of social and communicative developmental trajectories in preschoolers with autism spectrum disorders}

\author{
JEFFREY MUNSON, ${ }^{1,2}$ SUSAN FAJA, ${ }^{1,3}$ ANDREW MELTZOFF, ${ }^{3,4}$ ROBERT ABBOTT, $, 1,4$ \\ AND GERALDINE DAWSON, ${ }^{1-3}$ \\ ${ }^{1}$ University of Washington Autism Center and Center on Human Development and Disability, Seattle, Washington \\ ${ }^{2}$ Department of Psychiatry and Behavioral Sciences, University of Washington, Seattle, Washington \\ ${ }^{3}$ Department of Psychology, University of Washington, Seattle, Washington \\ ${ }^{4}$ Institute for Learning and Brain Sciences, Seattle, Washington \\ ${ }^{5}$ Department of Educational Psychology, University of Washington, Seattle, Washington \\ (Received December 1, 2007; Final Revision August 4, 2008; Accepted August 5, 2008)
}

\begin{abstract}
Currently, the heterogeneity in the developmental trajectories of autism spectrum disorders (ASD) is poorly understood. Preschool children with ASD participating in a longitudinal study received a battery of neurocognitive tasks that measured the learning of reward associations (Lrn-Rew), spatial working memory (SpatWM), and imitation from memory and novelty preference (Mem/Nov), as well as a measure of nonverbal problem-solving ability (NVDQ). Growth curve analyses via HLM were used to predict the variability in growth rates between age 4 to age 6.5 in Vineland Socialization and Communication scores. Individual differences in both Lrn-Rew and Mem/Nov were significantly related to Socialization and Communication growth rates above and beyond NVDQ, whereas SpatWM was not. Thus, specific aspects of neurocognitive functioning appear to be important predictors of developmental variability during the preschool years in children with ASD. We speculate that these findings support the combined role of ventromedial prefrontal and medial temporal lobe systems in the early pathogenesis of ASD and may be useful in predicting developmental trajectory. The benefits and challenges of assessing specific neurocognitive functions in children with autism is discussed with regard to general cognitive/developmental ability and the behavioral requirements of most assessment settings. (JINS, 2008, 14, 956-966.)
\end{abstract}

Keywords: Autistic disorder, Neuropsychology, Imitation, Language, Social adjustment, Longitudinal studies

\section{INTRODUCTION}

Autism is a neurodevelopmental disorder characterized by communication and social impairments as well as repetitive behaviors or interests. Within autism spectrum disorders (ASD) there is marked heterogeneity in symptom expression and developmental course. Understanding this variability, particularly during the preschool period when the diagnosis is commonly made and intervention begins, may have utility in identifying appropriate intervention strat-

Correspondence and reprint requests to: Jeffrey Munson, UW Autism Center, Box 357920, University of Washington, Seattle, WA 98195. E-mail: jeffmun@u.washington.edu egies and predicting response to treatment. Identification of neurocognitive predictors of functional outcome in autism is one approach toward understanding how variability in the development of specific brain circuits implicated in the etiology of autism spectrum disorders may contribute to the unfolding expression of symptoms.

Most longitudinal studies of children with ASD have found that early verbal and nonverbal abilities show positive correlations with subsequent language and cognitive outcome (Anderson et al., 2007; Charman et al., 2003, Gabriels et al., 2001; Mundy et al., 1990; Szatmari et al., 2003; Venter et al., 1992). Various measures of joint attention (such as pointing and alternating gaze) in preschoolers with autism have also shown positive relationships with later outcome 
(Anderson et al., 2007; Charman et al., 2003, Mundy et al., 1990; Sigman \& McGovern, 2005). However, a clear understanding of the relative contributions of these domains of functioning to outcome and the mechanisms of their effect has not yet emerged. Furthermore, the measures used in many investigations have been quite broad, and may miss specific neurocognitive abilities that could provide greater insight into neurobiological mechanisms underlying the skill development of children with autism. Neurobiological systems, particularly in the prefrontal cortex, medial temporal lobe, and cerebellum are presumed to play a major etiological role in autism. Several authors (e.g., Charman et al., 2005; Szatmari et al., 1989) therefore have suggested the importance of examining neurocognitive factors thought to reflect the integrity of specific brain systems implicated in the early neuropathology of autism as an important strategy in understanding the course of the disorder.

To date, few investigations of neurocognitive function in autism have been longitudinal and those investigating neurocognitive deficits in preschoolers with autism have provided mixed results. With preschoolers, aged 3 to 4 years, evidence of an autism-specific profile of impairment was not found on tasks tapping inhibition or working memory relative to mental age matched controls with developmental delay or typical development (Dawson et al., 2002; Griffith et al., 1999; Stahl \& Pry, 2002), although children with autism were nonetheless delayed relative to age-matched typical children in both IQ and neurocognitive function. However, in samples of slightly older children (mean age $=$ 5.4 years in both studies) children with ASD were impaired on tasks involving stimulus-reward association learning, flexibility, working memory and inhibition compared with developmentally delayed and typically developing children matched on mental age (Dawson et al., 1998; McEvoy et al., 1993). Individual variability in neurocognitive functioning and its concurrent relation to social skills, such as joint attention, has also been investigated. Performance on measures of early executive function (e.g., set-shifting, A-not-B, delayed response, spatial reversal, alternation) relates to joint attention in both children with autism and typical development (McEvoy et al., 1993; Mundy, 2003; Stahl \& Pry, 2002); however, this relationship has not been consistently replicated in children with autism (Griffith et al., 1999; Stahl \& Pry, 2002). Dawson et al. (2002) found a strong positive relation between tasks that measured learning reward associations and joint attention, whereas tasks such as the A-not-B and spatial reversal did not relate to joint attention in the same group of participants.

The goal of the current study is to understand the unique relationships between early neurocognitive function and later development of social and communicative behavior in preschoolers with autism. Multiple tasks were used to measure each of three domains, allowing a more sensitive assessment of children who may have more variable performance on any given task. The testing situation for each task was very similar requiring only limited verbal instructions and involving interaction with objects while the child was seated at a table across from the experimenter. Specifically, we selected tasks that measure (1) the ability to learn reward associations (Lrn-Rew) thought to reflect ventromedial prefrontal integrity (e.g., object discrimination reversal, rule learning in delayed nonmatch to sample); (2) spatial working memory (SpatWM) thought to reflect dorsolateral prefrontal integrity (e.g., A-not-B, spatial reversal); and (3) memory and preference for novelty (Mem/Nov) thought to reflect medial temporal lobe integrity (e.g., memory portion of the delayed nonmatch to sample, paired comparison, deferred imitation). Based on the Dawson et al. (2002) findings of a concurrent relationship between learning reward associations and joint social attention and the recent neurodevelopmental model of autism proposed by Bachevalier and Loveland (2006) emphasizing the role of frontal and medial temporal systems to account for the social and neurocognitive variability observed in children with ASD, we anticipated that the ability to learn reward associations during the preschool period would be more predictive of growth in adaptive behavior than would spatial working memory. In addition, memory and preference for novelty would also relate to subsequent social and communication growth independent of the contribution of reward-learning ability.

\section{METHODS}

\section{Participants}

Seventy children with autism spectrum disorders (58 boys, 12 girls) were included in the current study and were recruited from the greater Seattle area via local parent advocacy groups, hospitals, clinics, public schools, and the Department of Developmental Disabilities to participate in a larger longitudinal study of autism, developmental delay, and typical development. Exclusionary criteria included the presence of a neurological disorder of known etiology, significant sensory or motor impairment, major physical anomalies, history of serious head injury, and neurological disease. Assessments were conducted across 4-6 visits during the first assessment of the longitudinal study. Participants' ages ranged from 34 to 52 months $(M=43.5, S D=4.2)$ at the initial assessment. Diagnosis was made by clinicians at the University of Washington Autism Center using the Autism Diagnostic Interview (ADI-R; Lord et al., 1994) and Autism Diagnostic Observation Schedule-Generic (ADOS-G; Lord et al., 2000), which were integrated with clinical observation to make a diagnostic judgment based on DSM-IV criteria (American Psychiatric Association, 2000). The parent or legal guardian of each child provided written informed consent in compliance with the University of Washington Human Subjects Division.

Early cognitive development was assessed using the Mullen Scales of Early Learning (Mullen, 1995), a measure of language, perceptual, and motor abilities appropriate for infants through preschoolers. The Mullen yields scaled scores for Receptive Language, Expressive Language, Visual 
Table 1. Neurocognitive constructs and component variables

\begin{tabular}{|c|c|c|}
\hline Construct & Task & Variables \\
\hline \multirow[t]{4}{*}{ Lrn-Rew } & Delayed non-matching to sample (DNMS) & $\begin{array}{l}\text { Percent correct during rule-learning phase at } 5 \mathrm{~s} \text { for objects (up to } 20 \\
\text { trials) }\end{array}$ \\
\hline & Object discrimination reversal (ODR) & $\begin{array}{l}\text { Total percent of criteria met including the initial association and two } \\
\text { reversal criteria }\end{array}$ \\
\hline & & Number of errors during initial association (up to 25 trials) \\
\hline & & Number of perseverative errors during initial association \\
\hline \multirow[t]{4}{*}{ Mem/Nov } & Delayed non-matching to sample (DNMS) & $\begin{array}{l}\text { Percent correct at } 30 \mathrm{~s} \text { for objects if the initial criterion was met (10 } \\
\text { or } 15 \text { trials) }\end{array}$ \\
\hline & & Percent correct at $5 \mathrm{~s}$ for pictures ( 24 trials) \\
\hline & Paired Comparison (PC) & Percent of correct initial reaches (12 trails) \\
\hline & Deferred imitation (DI) & Percent correct imitation ( 5 actions) \\
\hline \multirow[t]{4}{*}{ SpatWM } & A-not-B (AnB) & Percent correct at $5 \mathrm{sec}$ (up to 24 trials) \\
\hline & & Percent correct at $12 \mathrm{sec}$ (up to 18 trials) \\
\hline & A-not-B with invisible displacement (AnB/ID) & Percent correct (up to 14 trials) \\
\hline & Spatial reversal (SR) & Percent correct ( 20 trials) \\
\hline
\end{tabular}

Note . Lrn-Rew $=$ Learning Reward Associations; Mem/Nov = Memory for Objects and Actions/Novelty Preference; SpatWM = Spatial Working Memory.

Reception, and Fine Motor subtests, as well as an overall Early Learning Composite. The Mullen subscales show good inter-scorer reliability, validity, and specificity (Mullen, 1995). At the initial assessment, the mean Mullen subtest T-scores were as follows: Visual Reception, $M=27.90$, $S D=11.92$, range $=20-61$; Fine Motor, $M=24.77, S D=$ 9.03, range $=20-59$; Receptive Language, $M=26.19, S D=$ 10.22 , range $=20-59$; Expressive Language, $M=26.14$, $S D=10.35$, range $=20-58$. A composite nonverbal ability score (NVDQ) was calculated by taking the average T-score on the Visual Reception and Fine Motor scales.

The Socialization and Communication domains of the Vineland Adaptive Behavior Scales (Sparrow et al., 1984) were administered to each child's primary caregiver semiannually, with an average of $6.6(S D=1.4$; range $=3-9)$ assessments between the ages of 36 and 78 months for each child in the sample. This number varied for individual children due to child's age at enrollment (i.e., children enrolled earlier had more follow-ups) and due to parental availability and willingness to complete follow-up assessments. Vineland age equivalence scores for the first assessment were as follows: Socialization, $M=18.20, S D=7.12$, range $=$ 6-40; Communication, $M=20.20, S D=10.20$, range $=$ $2-61$. The Vineland has good test-retest and inter-rater reliability. Study personnel were required to achieve 0.80 reliability with the lead Vineland administrator.

\section{Neurocognitive Tasks}

Testing was conducted during four to six 2- to 3-hour visits of which the neurocognitive tasks were a subset. Children were tested in the presence of a parent and breaks were provided as necessary. Children were praised for correct responses and food items or small toys were used for tasks requiring the child to search for hidden rewards. Children were only permitted to access these rewards when they responded correctly, with the exception of the paired comparison and deferred imitation tasks which involve no additional reward beyond the task objects. For tasks involving a forced choice, both stimuli were placed equidistant from the child. Three domains of neurocognitive function were assessed by averaging multiple measurements derived from tasks in three domains (see Table 1).

\section{Domain 1: learning reward associations (Lrn-Rew)}

Delayed nonmatching to sample (DNMS). For each trial, children were first presented with the sample (i.e., a novel junk object) obscuring a well containing a reward. The stimuli were hidden for a 5-s delay, while the sample was placed over an empty well and a nonmatching, novel object was placed over a second, baited well. Thus the child must learn that the reward is paired with the novel object. Then, both objects were presented. Children were told a treat had been hidden and were encouraged to look for it without additional directions. On incorrect reaches, correct stimulusreward pairings were shown. The correct side varied using a fixed order across trials. Children continued until they reached correctly for five consecutive trials or a maximum of 20 trials were administered (Diamond et al., 1999).

Object discrimination reversal. Rewards were hidden while a screen obscured the hiding locations. For the first trial, rewards were hidden inside wells with different objects (a plastic cat and dog) placed on top. The screen was lifted and children were encouraged to find the reward but given no additional instruction. The chosen object was noted, and for subsequent trials a reward was hidden only under the preferred object. The side of the baited object varied following a prespecified order. After children demonstrated an association between the object and reward by correctly reach- 
ing five times consecutively, a reversal occurred and children were required to learn that the reward was now under the opposite object. For incorrect reaches the reward was revealed while the experimenter said, "Look, it was over here." The task continued until two reversals were administered, or the child failed to reach criterion within 25 trials (Dawson et al., 2002).

\section{Domain 2: memory and novelty preference} (Mem/Nov)

Delayed nonmatching to sample (DNMS). If children met criterion with the 5-s delay, they were then administered either 10 or 15 additional trials with a 30 -s memory delay, depending on how quickly they met the initial criteria at a $5 \mathrm{~s}$ delay. In addition, a second version of the DNMS was administered in which photographs of objects were presented in plastic picture frames that covered the wells rather than the junk objects used in the first version. Children were administered 24 trials with a 5-s delay regardless of performance. Because they were administered after the initial rule learning phase of the DNMS, these portions of the task selectively emphasized memory by continuing to reward children for selecting the novel stimulus.

Visual paired-comparison memory. Children were presented with an unlabeled sample stimulus for $20 \mathrm{~s}$ and encouraged to attend to it. After the examination period, the object was removed for either $10 \mathrm{~s}$ or $3 \mathrm{~min}$. The sample and a novel object were then presented with no further directions. The object the child reached for first was scored as the child's preference. Children were expected to prefer the novel object, thus the task is thought to tap children's ability to remember and discriminate the sample from the novel object, but not the ability to learn stimulus-reward associations (Diamond, 1995). The task was presented during two different visits, for a total of 12 trials.

Deferred imitation memory. The imitation tasks were adapted from previous work on imitation in typical and atypical populations by Meltzoff (Meltzoff, 1988, 1999; Rast \& Meltzoff, 1995) and chosen to be sensitive to imitation abilities in the toddler-preschool developmental range. The task consisted of five actions on objects, such as banging blocks together, collapsing a transformable object, and touching one's head to a panel. After attracting the child's attention, the experimenter demonstrated each target act three times without verbal description of the actions or physical prompting. After all five actions were demonstrated, a 10-min memory delay was interposed during which the children left the test room. Then, the children returned and were presented with the test objects one at a time in their original order and told, "It's your turn. You can play with this."

\section{Domain 3: spatial working memory (SpatWM)}

A-not-B. Children observed as a reward was placed under one of two identical cups placed on the right and left side of the table. Following a brief obstruction by a screen, children were encouraged to find the reward but not given additional instructions. During the initial training phase, the screen obscured the cups for $3 \mathrm{~s}$ and children continued to the test phase only if they correctly retrieved the reward from both sides. During the testing phase a screen obscured the stimuli for $5 \mathrm{~s}$. Children were shown the correct location but not given the reward for incorrect searches. Rewards were hidden on the same side until two consecutive correct reaches were made, and then the reward was hidden on the opposite side (i.e., a reversal). After two reversals followed by two consecutive correct choices, the delay was increased to $12 \mathrm{~s}$ until children achieved 2 more reversals followed by two consecutive correct choices. Thus, the task requires working memory an inhibition of previous responses (Diamond, 1985). The task was discontinued either when the child progressed through all reversals or when 24 trials were administered.

A-not-B with invisible displacement. Children observed as a box with an open side facing the child was presented at the center of the table, and a reward was placed inside. A cover was draped across the open side, obscuring the reward from the child's view. While the child watched, the experimenter slid the box to the right or left. The box was then briefly obscured by a screen. During the delay an identical, but empty box was placed on the other side of the table, equidistant from the child. After the delay, the screen was lifted, and the child was prompted to find the reward. If the child reached incorrectly, the examiner showed the child the location of the reward, but the child was not permitted to retrieve the reward. As with the A-not-B task, the side of hiding was reversed after 2 consecutive correct trials and the task continued until three reversals with 2 consecutive correct trials following them or a maximum of 14 trials were administered (Diamond et al., 1997).

Spatial reversal. This task began with a practice trial, during which children were told, "I am hiding an \{object \}" as the examiner placed objects under identical cups to the right and left but behind a screen so the child could not see the hiding. The screen was lifted and children were encouraged to find the reward. For subsequent trials, the chosen side was noted and a single reward was hidden under the cup on the preferred side while obscured by a screen. Following incorrect trials the examiner said, "Let's try again." The child was not permitted to view or access the reward and no additional feedback was given before the screen was replaced and the next trial began. Over the course of 20 trials, the hiding side was reversed after every set of four consecutive correct trials (Kaufman et al., 1990).

\section{RESULTS}

\section{Statistical Procedure}

A series of Hierarchical linear models (HLM, Raudenbush et al., 2004) were used to predict variability in the individual linear growth trajectories of Vineland Socialization and 
Communication scores as a function of NVDQ, Mem/Nov, Lrn-Rew, and SpatWM performance measured at age 3-4 years. Each component variable was standardized across the sample $(M=0, S D=1)$, then averaged to create the neurocognitive composite variables (Mem/Nov, Lrn-Rew, and SpatWM, see Table 2 for descriptive statistics and intercorrelations of the predictor and component variables). To indicate the overall level of performance for the sample as a whole, the mean and $S D$ for the component variables are presented in their raw metric. The composite variables are simply the mean of each standardized component variable; thus the sample mean for the composite variables are near, but not exactly zero, and the $S D$ is consistently less than 1 (range $=.68-.79$ ). NVDQ scores were also standardized to have all level-2 predictors on the same metric to simplify interpretation of the model coefficients. In contrast to multivariate repeated-measures approaches, this approach explicitly models change within the individual while simultaneously testing whether other measured factors correlate with the variability in growth across individuals (Raudenbush et al., 2004). A linear function was fit for each child's Vineland age equivalence scores resulting in two random-effect parameters (intercept or the child's estimated Vineland age equivalence score at 48 months, and slope or rate of change across the time span) which describe the course of development in social and communication ability. The child's age was centered at 48 months so the intercept in the model can be interpreted as the sample mean when the children were 4 years old.

\section{Unconditional Growth Model}

The unconditional growth model for Vineland Socialization and Communication scores showed a mean Socialization age equivalence of 20.75 at 48 months (intercept) and a mean growth rate of 0.557 age equivalence points/month (slope; Table 3, Model 1). Vineland Communication scores showed a mean age equivalence of 22.47 (intercept) at 48 months and a mean growth rate of 0.727 age equivalence points/month (slope). Reliabilities for these growth parameters were high (Socialization: intercept $=0.71$, slope $=$ 0.64; Communication: intercept $=0.91$, slope $=0.83$ ) and these parameters showed significant variability across the sample. The intercept and slope parameters were correlated for both Vineland scores (Socialization: $\tau=0.56$; Communication $\tau=0.73$ ). As seen in Figure 1, the correlation between initial status and growth rate produces a fan spread of the estimated growth trajectories such that children with higher Vineland scores at the beginning of the study tended to increase at faster rates, particularly for the communication scores. Thus, the sample as a whole, showed significant delays in Socialization and Communication at 48 months and lower than normal rates of growth (Socialization increasing 0.557 age-equivalence points/month, Communication increasing 0.727 age-equivalence points/month). The social and communication intercept terms correlated 0.70 and slope terms 0.82 .

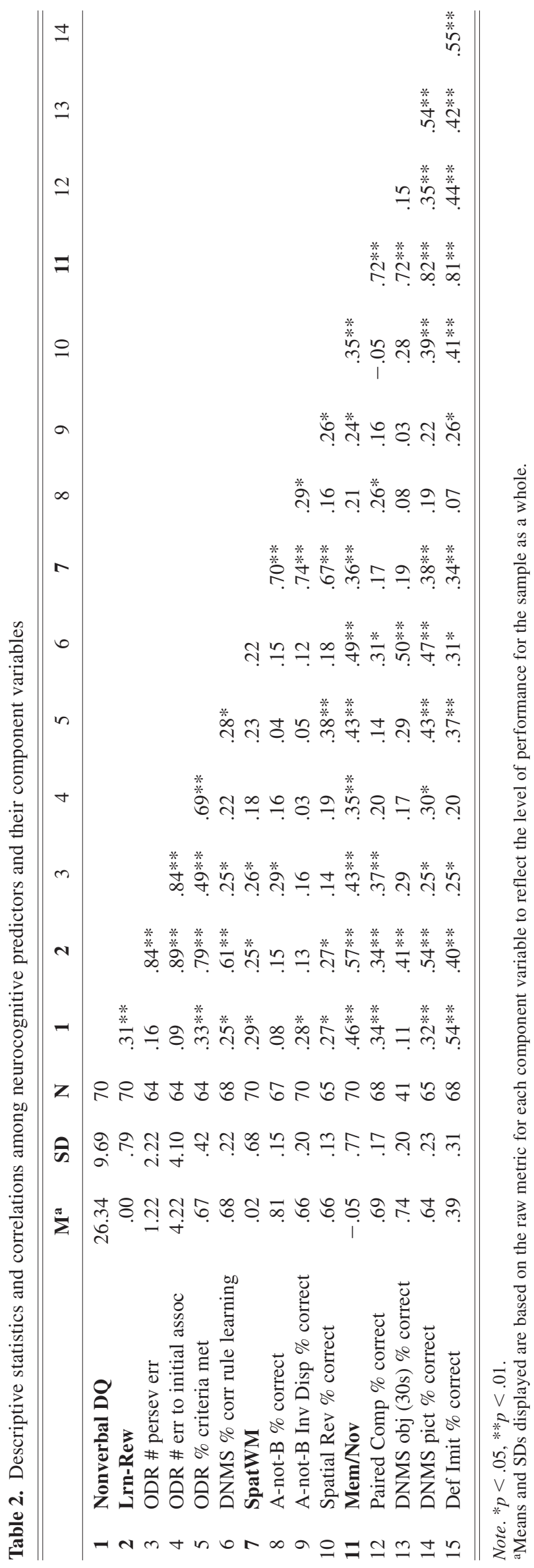


Table 3. HLM model of growth in Vineland Socialization and Communication age equivalence scores: Model 1, Unconditional model; Model 2, NVDQ as predictor

\begin{tabular}{|c|c|c|c|c|c|c|}
\hline & \multicolumn{3}{|c|}{ Vineland Socialization } & \multicolumn{3}{|c|}{ Vineland Communication } \\
\hline & coeff & SE & Variance & coeff & SE & Variance \\
\hline \multicolumn{7}{|c|}{ Model 1: Unconditional model } \\
\hline \multicolumn{7}{|c|}{ Intercept (at 48 mos.) } \\
\hline Constant & $20.89 * *$ & 0.94 & $45.11 * *$ & $22.45 * *$ & 1.23 & $97.34 * *$ \\
\hline \multicolumn{7}{|c|}{ Slope (growth rate) } \\
\hline Constant & $0.534 * *$ & 0.055 & $0.159 * *$ & $0.726 * *$ & 0.059 & $0.210 * *$ \\
\hline \multicolumn{7}{|c|}{ Model 2: NVDQ as predictor } \\
\hline \multicolumn{7}{|c|}{ Intercept (at 48 mos.) } \\
\hline Constant & $20.88 * *$ & 0.92 & $41.84 * *$ & $22.49 * *$ & 0.94 & $52.51 * *$ \\
\hline NVDQ & $1.87 *$ & 0.83 & & $6.70 * *$ & 0.99 & \\
\hline \multicolumn{7}{|c|}{ Slope (growth rate) } \\
\hline Constant & $0.533 * *$ & 0.049 & $0.114 * *$ & $0.724 * *$ & 0.053 & $0.169 * *$ \\
\hline NVDQ & $0.217 * *$ & 0.049 & & $0.204 * *$ & 0.045 & \\
\hline
\end{tabular}

Note. $* p<.05, * * p<.001$.

\section{Relationships Between NVDQ, Lrn-Rew, Mem/Nov, and SpatWM with Social and Communication Growth}

We began the analyses by first examining the relationship of NVDQ and Vineland growth (Table 3, Model 2). NVDQ was significantly, positively related to both Socialization and Communication values at 48 months as well as subsequent growth rates over the next 2-3 years. NVDQ accounted for $28.3 \%$ [(0.159-0.114)/0.159] and $19.5 \%[(0.210-0.169) / 0.210]$ of the variance in the Socialization and Communication slope parameters, respectively [(slope parameter variance model $1_{1}$ - slope parameter variance $_{\text {model } 2}$ )/slope parameter variance model $1_{1}$ ].

Next, each neurocognitive predictor was examined with NVDQ to assess whether they accounted for any additional variability in growth beyond the measure of general nonverbal abilities (Table 4). NVDQ was significantly related to concurrent Communication but not Socialization scores (intercept term). The relationship between Mem/ Nov and the Communication intercept indicated the only time a neurocognitive variable related to the concurrent level of functioning.

However, Both Lrn-Rew and Mem/Nov were significantly related to Social and Communication growth rates, whereas SpatWM was not. ${ }^{1}$ Thus, Lrn-Rew was related to later growth in Socialization and Communication scores beyond NVDQ rather than having a unique contribution to concurrent levels of these skills and Mem/Nov related to later growth, but not concurrent levels, of Socialization scores.

${ }^{1}$ To account for potential ceiling effects from the A-not-B component variable, which had the highest mean score and is a developmentally simpler task, this variable was removed from the SpatWM construct and the analysis repeated. Again, no significant relationship between SpatWM and Vineland growth parameters was found (all $t$ 's $\leq 1.73, p$ 's $>.05$ ).
Final models were then run that included NVDQ, LrnRew, and Mem/Nov together as the predictors to assess the relative contributions of Lrn-Rew and Mem/Nov on growth rates Table 5). No single variable was significantly related to the Socialization intercept in this combined model. However, NVDQ, Lrn-Rew, and Mem/Nov each continued to show unique, significant relationships with Socialization growth. This final model accounts for a total of $52.2 \%$ of the variance in Socialization growth.

For Communication, NVDQ was again the only variable showing a significant concurrent relationship at 48 months. NVDQ and Lrn-Rew remained a significant predictor of Communication growth, whereas Mem/Nov fell just short of significance. This final model accounts for a total of $42.4 \%$ of the variance in Communication growth.

To illustrate these results, we can compare hypothetical children who score uniformly at $-1,0$, and 1 SD from the sample mean on NVDQ, Lrn-Rew, and Mem/Nov. These children would have estimated growth rates of .097, .544, and 0.991 Socialization age equivalence points/month and $.279, .734$, and 1.189 Communication age equivalence points/month, respectively.

\section{DISCUSSION}

The current study followed the developmental trajectory of 70 preschool children with autism spectrum disorders and examined the degree to which performance on tasks involving learning reward associations (Lrn-Rew; ODR and DNMS rule-learning component), memory and novelty preferences (Mem/Nov; DNMS memory component, visual paired comparison, deferred imitation), and spatial working memory (SpatWM; A-not-B and spatial reversal) add to the prediction of the rate of social and communication skill acquisition beyond that accounted for by general nonverbal developmental quotient (NVDQ). These tasks were chosen 

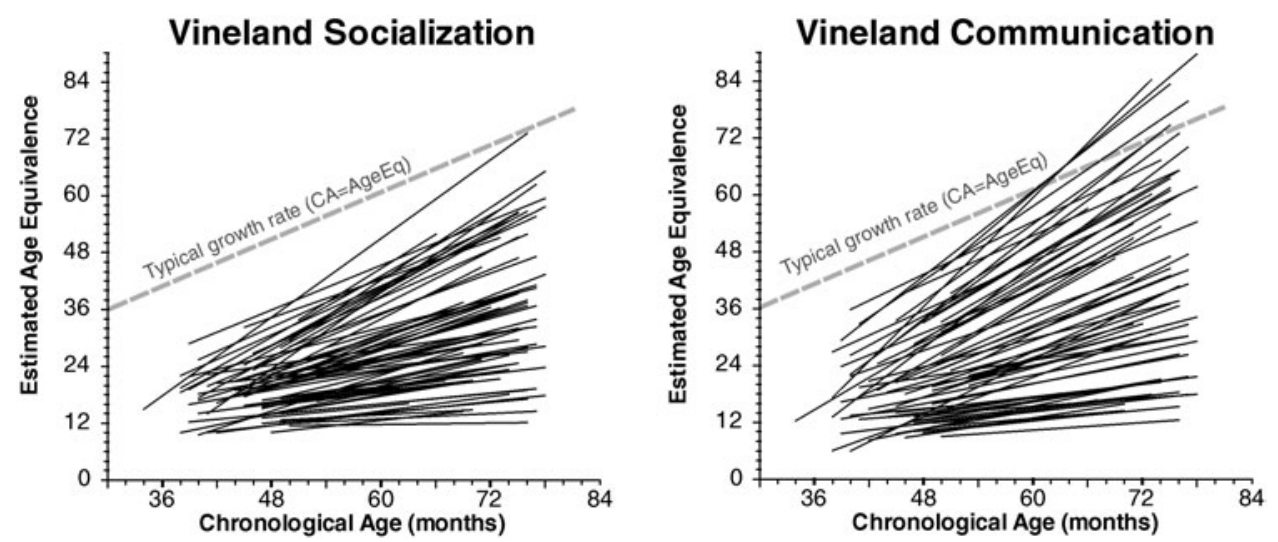

Fig. 1. Plots of estimated Vineland growth trajectories from the HLM unconditional model.

given the evidence from adult lesion studies and animal models, linking them to the functional integrity of the ventromedial prefrontal cortex (Lrn-Rew) (Butter, 1969; Butters et al., 1973; Jones \& Mishkin, 1972; Kowalska et al.,
1991; Mishkin, 1964; Rolls et al., 1994), dorsolateral prefrontal cortex (SpatWM) (e.g., Diamond \& GoldmanRakic, 1986, 1989; Goldman et al., 1970), and medial temporal lobe (Mem/Nov) (McDonough et al., 1995; Melt-

Table 4. HLM model of growth in Vineland Socialization and Communication age equivalence scores as a function of NVDQ and a single neurocognitive predictor

\begin{tabular}{|c|c|c|c|c|c|c|}
\hline & \multicolumn{3}{|c|}{ Vineland Socialization } & \multicolumn{3}{|c|}{ Vineland Communication } \\
\hline & coeff & SE & Variance & coeff & SE & Variance \\
\hline \multicolumn{7}{|c|}{ NVDQ and Lrn-Rew } \\
\hline \multicolumn{7}{|c|}{ Intercept (48 mos.) } \\
\hline Constant & $20.91 * * *$ & 0.91 & $40.90 * * *$ & $22.52 * * *$ & 0.92 & $50.20 * * *$ \\
\hline NVDQ & $1.76+$ & 0.89 & & $6.25 * * *$ & 1.04 & \\
\hline Lrn-Rew & 0.48 & 0.96 & & $1.87+$ & 1.04 & \\
\hline \multicolumn{7}{|c|}{ Slope (growth rate) } \\
\hline Constant & $0.532 * * *$ & 0.044 & $0.086 * * *$ & $0.725 * * *$ & 0.048 & $0.131 * * *$ \\
\hline NVDQ & $0.158 * *$ & 0.051 & & $0.138 * *$ & 0.044 & \\
\hline Lrn-Rew & $0.232 * * *$ & 0.039 & & $0.264 * * *$ & 0.046 & \\
\hline \multicolumn{7}{|c|}{ NVDQ and SpatWM } \\
\hline \multicolumn{7}{|c|}{ Intercept (48 mos.) } \\
\hline Constant & $20.86 * * *$ & 0.91 & $41.44 * * *$ & $22.45 * * *$ & 0.92 & $51.31 * * *$ \\
\hline NVDQ & $1.68+$ & 0.86 & & $6.37 * * *$ & 1 & \\
\hline SpatWM & 0.94 & 1.21 & & 1.69 & 1.3 & \\
\hline \multicolumn{7}{|c|}{ Slope (growth rate) } \\
\hline Constant & $0.532 * * *$ & 0.049 & $0.11 * * *$ & $0.721 * * *$ & 0.052 & $0.160 * * *$ \\
\hline NVDQ & $0.200 * * *$ & 0.049 & & $0.175^{* *}$ & 0.047 & \\
\hline SpatWM & 0.085 & 0.073 & & $0.146+$ & 0.078 & \\
\hline \multicolumn{7}{|c|}{ NVDQ and Mem/Nov } \\
\hline \multicolumn{7}{|c|}{ Intercept (48 mos.) } \\
\hline Constant & $20.96 * * *$ & 0.93 & $39.67 * * *$ & $22.63 * * *$ & 0.93 & $48.46 * * *$ \\
\hline NVDQ & 1.02 & 0.93 & & $5.65 * * *$ & 1.11 & \\
\hline $\mathrm{Mem} / \mathrm{Nov}$ & $2.30+$ & 1.31 & & $2.92 *$ & 1.29 & \\
\hline \multicolumn{7}{|c|}{ Slope (growth rate) } \\
\hline Constant & $0.550 * * *$ & 0.045 & $0.086 * * *$ & $0.740 * * *$ & 0.050 & $0.137 * * *$ \\
\hline NVDQ & $0.129 *$ & 0.053 & & $0.111^{*}$ & 0.051 & \\
\hline $\mathrm{Mem} / \mathrm{Nov}$ & $0.254 * * *$ & 0.058 & & $0.267 * * *$ & 0.070 & \\
\hline
\end{tabular}

Note. $* p<.05, * * p<.01, * * * p<.001$. 
Table 5. HLM model of growth in Vineland Socialization and Communication age equivalence scores as a function of NVDQ, Lrn-Rew, and Mem/Nov

\begin{tabular}{|c|c|c|c|c|c|c|}
\hline & \multicolumn{3}{|c|}{ Vineland Socialization } & \multicolumn{3}{|c|}{ Vineland Communication } \\
\hline & coeff & SE & Variance & coeff & SE & Variance \\
\hline \multicolumn{7}{|c|}{ Intercept (48 mo) } \\
\hline Constant & $21.01 * * *$ & 0.92 & $38.91 * * *$ & $22.63 * * *$ & 0.91 & $48.21 * * *$ \\
\hline NVDQ & 1.15 & 0.93 & & $5.67 * * *$ & 1.10 & \\
\hline Lrn-Rew & -0.72 & 1.25 & & 0.74 & 1.35 & \\
\hline Mem/Nov & 2.52 & 1.64 & & 2.40 & 1.66 & \\
\hline \multicolumn{7}{|c|}{ Slope (growth rate) } \\
\hline Constant & $0.544 * * *$ & 0.043 & $0.076^{* * *}$ & $0.734 * * *$ & 0.047 & $0.121 * * *$ \\
\hline NVDQ & $0.116^{*}$ & 0.053 & & $0.098^{*}$ & 0.048 & \\
\hline Lrn-Rew & $0.153 * *$ & 0.052 & & $0.188 * *$ & 0.066 & \\
\hline Mem/Nov & $0.178 *$ & 0.071 & & $0.169^{+}$ & 0.090 & \\
\hline
\end{tabular}

Note. ${ }^{+} p<.10, * p<.05, * * p<.01, * * * p<.001$.

zoff, 1990; Zola et al., 2000; Zola-Morgan \& Squire, 1993); each of which has been implicated in the development of autism and the individual differences observed among these children.

Growth curve analyses using HLM indicated that nonverbal problem-solving ability, the ability to learn reward associations, and memory and novelty preference measured at roughly 4 years of age each significantly contributed to the predicted rate of Vineland Socialization growth between ages 4 and 6.5 years and accounting for $52.2 \%$ of the variability in this growth. For Vineland Communication growth, a similar pattern for the neurocognitive factors was observed, accounting for $42.4 \%$ of the variability; however, memory and novelty preference fell just short of significance. In contrast to reward learning and memory and novelty preference, spatial working memory did not add to the prediction of growth beyond NVDQ during this age period. These findings provide support for our hypothesis that measures of reward learning in the preschool period predict the rate of social and communication growth between ages 4 and $6 \frac{1}{2}$ beyond that accounted for by NVDQ. The unique role of memory ability and novelty preference in the prediction of social and communication growth was similarly supported. Memory and novelty preference significantly predicted communication growth beyond NVDQ when only those two predictors were in the model. However, in the full model that included NVDQ and reward learning, the memory and novelty preference factor relationship with communication growth fell just short of statistical significance $(p<.10)$.

The current findings suggest some specificity in the relationship between neurocognitive factors measured in preschool and the developmental trajectory of socialization and communication abilities in young children with autism spectrum disorders. This predictive relationship was seen above and beyond the nonverbal abilities as measured by the Mullen Scales of Early Learning. Similarly, Dawson et al. (2002) demonstrated in preschoolers with ASD that a composite of tasks related to memory, novelty preference and reward learn- ing showed a concurrent relationship to joint attention, a fundamental social communication skill, in preschoolers with autism, while spatial working memory tasks did not. Thus, our current finding that these factors related to social and communication growth is consistent with previous work, and extends these findings by demonstrating their utility as predictive factors.

One interpretation of these results is that better performance on reward learning and novelty preference tasks reflect a greater likelihood that the child will detect and learn the complex interpersonal contingencies present in their social environment. Extracting these relevant information patterns requires flexibility in the face of ever changing stimulus-reward pairings that implicitly vary across people and social contexts and rapidly shift between sensory modalities. Perseveration on familiar and predictable objects may lead to a failure to attend to and apprehend available social and communicative information. Similarly, children may focus on only one aspect of the social environment, such as the multimodal perceptual stimulation conveyed by the mouth of a speaker, while neglecting information contained in the speaker's eyes. This type of difficulty may contribute to the observed deficits in ability to follow gaze (i.e., joint attention).

One strength of this study is the use of multiple measures of each neurocognitive domain drawn from tasks that are administered in the same context (child and experimenter seated at a table) using similar materials. These very specific tasks were examined in growth curve models along with NVDQ, a presumably broader construct reflecting nonverbal problem solving. It is interesting to note that of all the specific neurocognitive component variables, deferred imitation correlated with NVDQ, $r=.63$, whereas all other variables correlated from .15 to .39 . The NVDQ variable used in the study is derived from the Mullen Visual Reception and Fine Motor subscales. There are 42 items on these subscales beyond the 9-month age equivalence level, 22 (52\%) of which direct the administrator to physically dem- 
onstrate the desired behavior to the child and another 10 (24\%) of which involve the administrator pointing to an object or picture or tracing with their finger during item administration. Thus, three-fourths of the items beyond early infancy used to assess the nonverbal problem-solving abilities on the Mullen involve behavioral copying and/or pointing to some degree; two areas in which children with autism spectrum disorders show consistent difficulties. The use of IQ or overall developmental level as a covariate in children with autism must be done thoughtfully. Limitations in basic skills like joint attention, which have long been mastered by most preschool children, may hamper the child with autism's ability to perform a given item. Continued work in this area would benefit from explicit measurement of gaze during neurocognitive assessment to determine how limitations in the appropriate direction of visual attention may impact performance.

During cognitive assessment the child is presumably simultaneously engaged in a wide array of social-cognitive processes while the child shares an interaction with the experimenter focused on the test materials. Although elements of social-cognition are an implicit part of the assessment context, the current findings suggest individual differences on certain neurocognitive variables (learning reward associations, memory, and preference for novelty) and not others (spatial working memory) were related to subsequent social and communication growth in this period. Given that all tasks involved the examiner acting on objects while the child watches, it is unlikely that these common social factors account for the specificity of these findings.

Our findings are of interest given current developmental models of executive function and models of aberrant development of related brain regions in children with autism. Cunningham and Zelazo (2007) proposed a model unifying the affective and cognitive elements of evaluative processing and highlighting the roles of the prefrontal cortex and limbic systems. Their model emphasizes the progression from rapid emotional or automatic evaluations (e.g., simple stimulus-reward rules) to more reflective, high-order processing through the iterative reprocessing of relevant information via hierarchy of neural networks. Over the course of development, the capacity for more complex representations comes on line following earlier development of the affective elements in the system resulting in greater sophistication and flexibility in problem solving and decision making behavior. It has been proposed that autism is primarily a disorder of the affective portions of this system (i.e., tagging stimuli, forming, and modifying reward associations), which in turn impacts later development of more purely cognitive executive functions (e.g., working memory, planning) performed by relatively lateral aspects of the prefrontal cortex (Zelazo \& Müller, 2002). This model compliments Bachevalier and Loveland's (2006) emphasis on frontal and medial temporal systems as playing a role in the social and neurocognitive variability observed in children with ASD.

This study is limited in that it did not examine the functional role of other regions possibly involved in the devel- opment of autism symptoms (e.g., cerebellum, basal ganglia structures, etc.). Thus the relative importance of the domains measured in the current study should be further evaluated in the context of these other systems. In addition, as the neurocognitive predictors were constructed on a theoretical basis, the intercorrelation of the component variables varied and was lowest among the spatial working memory construct. It is possible that this impacted the likelihood of spatial working memory emerging as a significant correlate of growth. Detailed analysis of specific variables within a large neurocognitive battery is difficult as many tasks simply result in missing data when a child is unable to complete a baseline set of trials. Methods for handling missing data at level 2 in a two-level hierarchical model have been recently proposed (Shin \& Raudenbush, 2007) but not yet widely implemented. Another limitation is the fact that developmental outcome was assessed only using the Vineland. Continued work in this area should add repeated observational measures of behavior to supplement parent report. We are currently following a different sample of preschoolers with autism spectrum disorders participating in a randomized clinical trial of a multifaceted intensive behavioral intervention. Similar measures of neurocognitive function are being assessed to examine how they may influence responsiveness to treatment.

\section{ACKNOWLEDGMENTS}

This research was supported by the National Institute of Child Health and Human Development (U19HD34565, P50HD066782) and the National Institute of Mental Health (U54MH066399) and the Cure Autism Now Foundation.

\section{REFERENCES}

American Psychiatric Association. (2000). Diagnostic and Statistical Manual of Mental Disorders, Revised 4th edition. Washington, DC: American Psychiatric Association Press.

Anderson, D.K., Lord, C., Risi, S., DiLavore, P.S., Shulman, C., Thurm, A., Welch, K., \& Pickles, A. (2007). Patterns of growth in verbal abilities among children with autism spectrum disorder. Journal of Consulting and Clinical Psychology, 75, 594-604.

Bachevalier, J. \& Loveland, K.A. (2006). The orbitofrontalamygdala circuit and self-regulation of social-emotional behavior in autism. Neuroscience and Biobehavioral Reviews, 30, 97-117.

Butter, C.M. (1969). Perseveration in extinction and in discrimination reversal tasks following selective frontal ablations in Macaca mulatta. Physiology and Behavior, 4, 163-171.

Butters, N., Butter, C., Rosen, J., \& Stein, D. (1973). Behavioral effects of sequential and one-stage ablations of orbital prefrontal cortex in the monkey. Experimental Neurology, 39, 204-214.

Charman, T., Baron-Cohen, S., Swettenham, J., Baird, G., Drew, A., \& Cox, A. (2003). Predicting language outcome in infants with autism and pervasive developmental disorder. International Journal of Language and Communication Disorders, $38,265-285$. 
Charman, T., Taylor, E., Drew, A., Cockerill, H., Brown, J.A., \& Baird, G. (2005). Outcome at 7 years of children diagnosed with autism at age 2: Predictive validity of assessments conducted at 2 and 3 years of age and pattern of symptom change over time. Journal of Child Psychology and Psychiatry, 46, 500-513.

Cunningham, W.A. \& Zelazo, P.D. (2007). Attitudes and evaluations: A social cognitive neuroscience perspective. Trends in Cognitive Sciences, 11, 97-104.

Dawson, G., Meltzoff, A.N., Osterling, J., \& Rinaldi, J. (1998). Neuropsychological correlates of early symptoms of autism. Child Development, 69, 1276-1285.

Dawson, G., Munson, J., Estes, A., Osterling, J., McPartland, J., Toth, K., Carver, L., \& Abbott, R. (2002). Neurocognitive function and joint attention ability in young children with autism spectrum disorder versus developmental delay. Child Development, 73, 345-358.

Diamond, A. (1985). Development of the ability to use recall to guide action, as indicated by infants' performance on A-not-B. Child Development, 56, 868-883.

Diamond, A. (1995). Evidence for robust recognition memory early in life even when assessed by reaching behavior. Journal of Experimental Child Psychology, 59, 419-456.

Diamond, A., Churchland, A., Cruess, L., \& Kirkham, N.Z. (1999). Early developments in the ability to understand the relation between stimulus and reward. Developmental Psychology, 35, 1507-1517.

Diamond, A. \& Goldman-Rakic, P.S. (1986). Comparative development in human infants and infant rhesus monkeys of cognitive functions that depend on prefrontal cortex. Society for Neuroscience Abstracts, 12, 742.

Diamond, A. \& Goldman-Rakic, P.S. (1989). Comparison of human infants and rhesus monkeys on Piaget's AB task: Evidence for dependence on dorsolateral prefrontal cortex. Experimental Brain Research, 74, 24-40.

Diamond, A., Prevor, M.B., Callender, G., \& Druin, D.P. (1997). Prefrontal cortex cognitive deficits in children treated early and continuously for PKU. Monographs of the Society for Research in Child Development, 62, (4, Serial No. 205).

Gabriels, R.L., Hill, D.E., Pierce, R.A., Rogers, S.J., \& Wehner, B. (2001). Predictors of treatment outcome in young children with autism: A retrospective study. Autism, 5, 407-429.

Goldman, P.S., Rosvold, H.E., \& Mishkin, M. (1970). Evidence for behavioral impairment following prefrontal lobectomy in the infant monkey. Journal of Comparative Physiological and Psychology, 70, 454-463.

Griffith, E.M., Pennington, B.F., Wehner, E.A., \& Rogers, S.J. (1999). Executive functions in young children with autism. Child Development, 70, 817-832.

Jones, B. \& Mishkin, J. (1972). Limbic lesions and the problem of stimulus-reinforcement associations. Experimental Neurology, 36, 362-377.

Kaufman, P., Leckman, J., \& Ort, S. (1990). Delayed response performance in males with Fragile X. Journal of Clinical and Experimental Neuropsychology, 12, 69.

Kowalska, D., Bachevalier, J., \& Mishkin, M. (1991). The role of the inferior prefrontal convexity in performance of delayed nonmatching-to-sample. Neuropsychologia, 29, 583-600.

Lord, C., Risi, S., Lambrecht, L., Cook, E.H., Jr., Leventhal, B.L., DiLavore, P.C., Pickles, A., \& Rutter, M. (2000). The Autism Diagnostic Observation Schedule-Generic: A standard measure of social and communication deficits associated with the spectrum of autism. Journal of Autism and Developmental Disorders, 30, 205-223.

Lord, C., Rutter, M., \& LeCouteur, A. (1994). Autism diagnostic interview-revised: A revised version of a diagnostic interview for caregivers of individuals with possible pervasive developmental disorders. Journal of Autism and Developmental Disorders, 24, 659-685.

McDonough, L., Mandler, J.M., McKee, R.D., \& Squire, L.R. (1995). The deferred imitation task as a nonverbal measure of declarative memory. Proceedings of the National Academy of Sciences, 92, 7580-7584.

McEvoy, R., Rogers, S.J., \& Pennington, B.F. (1993). Executive function and social communication deficits in young autistic children. Journal of Child Psychology and Psychiatry, 34, 563-578.

Meltzoff, A.N. (1988). Infant imitation after a 1-week delay: Longterm memory for novel acts and multiple stimuli. Developmental Psychology, 24, 470-476.

Meltzoff, A.N. (1990). Towards a developmental cognitive science: The implications of cross-modal matching and imitation for the development of representation and memory in infancy. In A. Diamond (Ed.), Annals of the New York Academy of Sciences, Vol. 608: The development and neural bases of higher cognitive functions (pp. 1-37). New York: New York Academy of Sciences.

Meltzoff, A.N. (1999). Origins of theory of mind, cognition and communication. Journal of Communication Disorders, 32, 251-269.

Mishkin, M. (1964). Perseveration of central sets after frontal lesions in monkeys. In J.M. Warren \& K. Akert (Eds.), The Frontal Granular Cortex and Behavior (pp. 219-241). New York: McGraw-Hill.

Mullen, P. (1995). Mullen Scales of Early Learning, AGS Edition. Bloomington, MN: Pearson's Assessment Group.

Mundy, P. (2003). Annotation: The neural basis of social impairments in autism: The role of the dorsal medial-frontal cortex and anterior cingulate system. Journal of Child Psychology and Psychiatry, 44, 793-809.

Mundy, P., Sigman, M., \& Kasari, C. (1990). A longitudinal study of joint attention and language development in autistic children. Journal of Autism and Developmental Disorders, 20, 115-128.

Rast, M. \& Meltzoff, A.N. (1995). Memory and representation in young children with Down syndrome: Exploring deferred imitation and object permanence. Development and Psychopathology, 3, 393-407.

Raudenbush, S., Bryk, A., Cheong, Y., \& Congdon, R. (2004). HLM6: Hierarchical Linear and Nonlinear Modeling. Lincolnwood, IL: Scientific Software International.

Rolls, E.T., Hornak, J., Wade, D., \& McGrath, J. (1994). Emotionrelated learning in patients with social and emotional changes associated with frontal lobe damage. Journal of Neurology, Neurosurgery, and Psychiatry, 57, 1518-1524.

Shin, Y. \& Raudenbush, S.W. (2007). Just-identified versus overidentified two-level hierarchical linear models with missing data. Biometrics, 63, 1262-1268.

Sigman, M. \& McGovern, C. (2005). Improvement in cognitive and language skills from preschool to adolescence in autism. Journal of Autism and Developmental Disorders, 35, 15-23.

Sparrow, S.S., Balla, D.A., \& Cicchetti, D. (1984). Vineland Adaptive Behavior Scales. Circle Pines, MN: American Guidance Service.

Stahl, L. \& Pry, R. (2002). Joint attention and set-shifting in young children with autism. Autism, 6, 383-396. 
Szatmari, P., Bartolucci, G., Bremner, R., Bond, S., \& Rich, S. (1989). A follow-up study of high-functioning autistic children. Journal of Autism and Developmental Disorders, 19, 213-225.

Szatmari, P., Bryson, S.E., Boyle, M.H., Streiner, D.L., \& Duku, E. (2003). Predictors of outcome among high functioning children with autism and Asperger syndrome. Journal of Child Psychology and Psychiatry, 44, 520-528.

Venter, A., Lord, C., \& Schopler, E. (1992). A follow-up study of high-functioning autistic children. Journal of Child Psychology and Psychiatry, 33, 489-507.
Zelazo, P.D. \& Müller, U. (2002). The balance beam in the balance: Reflections on rules, relational complexity, and developmental processes. Journal of Experimental Child Psychology, $81,458-465$

Zola, S.M., Squire, L.R., Teng, E., Stefanacci, L., Buffalo, E.A., \& Clark, R.E. (2000). Impaired recognition memory in monkeys after damage limited to the hippocampal region. The Journal of Neuroscience, 20, 451-463.

Zola-Morgan, S. \& Squire, L.R. (1993). Neuroanatomy of memory. Annual Review of Neuroscience, 6, 547-563. 